Bull. Korean Math. Soc. 49 (2012), No. 2, pp. 353-358

http://dx.doi.org/10.4134/BKMS.2012.49.2.353

\title{
A NOTE ON PSEUDO-RIEMANNIAN ASSOCIATIVE FERMIONIC NOVIKOV ALGEBRAS
}

\author{
Zhiqi Chen and Fuhai Zhu
}

\begin{abstract}
In this paper, we focus on pseudo-Riemannian associative fermionic Novikov algebras. We prove that the underlying Lie algebras of pseudo-Riemannian associative fermionic Novikov algebras are 2-step nilpotent and that pseudo-Riemannian associative fermionic Novikov algebras are 3-step nilpotent. Moreover, we construct a pseudo-Riemannian associative fermionic Novikov algebra in dimension 14, which is not a Novikov algebra. It implies that the inverse proposition of Corollary 2 in the paper "Pseudo-Riemannian Novikov algebras" [J. Phys. A: Math. Theor. 41 (2008), 315207] does not hold.
\end{abstract}

\section{Underlying Lie algebras of pseudo-Riemannian associative fermionic Novikov algebras}

$\mathrm{Gel}^{\prime}$ fand and Dikii gave a bosonic formal variational calculus in [9, 10] and $\mathrm{Xu}$ gave a fermionic formal variational calculus in [15]. Moreover, motivated by the super-symmetric theory, a formal variational calculus of super-variables was given by $\mathrm{Xu}$ in [16] which combines the bosonic theory of Gel'fand-Dikii and the fermionic theory. Fermionic Novikov algebras are related to the Hamiltonian super-operator in terms of this theory. A fermionic Novikov algebra $A$ is a vector space over a field $\mathbb{F}$ with a bilinear product $(x, y) \mapsto x y$ satisfying

$$
(x y) z-x(y z)=(y x) z-y(x z),
$$

$$
(x y) z=-(x z) y
$$

for any $x, y, z \in A$. It corresponds to the following Hamiltonian operator $H$ of type $0[16]$ :

$$
H_{\alpha, \beta}^{0}=\sum_{\gamma \in I}\left(a_{\alpha, \beta}^{\gamma} \Phi_{\gamma}(2)+b_{\alpha, \beta}^{\gamma} \Phi_{\gamma} D\right), \quad a_{\alpha, \beta}^{\gamma}, b_{\alpha, \beta}^{\gamma} \in \mathbb{R} .
$$

Received November 2, 2010; Revised September 6, 2011.

2010 Mathematics Subject Classification. 17B60, 17A30, 17D25.

Key words and phrases. Novikov algebra, fermionic Novikov algebra, pseudo-Riemannian Lie algebra. 
Fermionic Novikov algebras are a special class of left-symmetric algebras which only satisfy equation (1.1). Left-symmetric algebras are a class of non-associative algebras arising from the study of affine manifolds, affine structures and convex homogeneous cones $[4,14]$. The commutator of a left-symmetric $A$

$$
[x, y]=x y-y x
$$

defines a Lie algebra, which is called the underlying Lie algebra of $A$.

A pseudo-Riemannian connection is a pseudo-metric connection such that the torsion is zero and parallel translations preserve the bilinear form on the tangent spaces [13]. The corresponding structure on a fermionic Novikov algebra $A$ is a non-degenerate symmetric bilinear form $f: A \times A \rightarrow \mathbb{F}$ such that

$$
f(x y, z)+f(y, x z)=0 \quad \text { for any } x, y, z \in A .
$$

Such a fermionic Novikov algebra is called a pseudo-Riemannian fermionic Novikov algebra. It is given in [18] that the underlying Lie algebra of a pseudoRiemannian fermionic Novikov algebra is a pseudo-Riemannian Lie algebra. A Lie algebra $\mathfrak{g}$ over a field $\mathbb{F}$ is called a pseudo-Riemannian Lie algebra if there is a bilinear product $(x, y) \mapsto x y$ such that, for any $x, y, z \in \mathfrak{g}$,

$$
x y-y x=[x, y], \quad[x y, z]+[x, z y]=0
$$

and a non-degenerate symmetric bilinear form $($,$) on \mathfrak{g}$ such that

$$
(x y, z)+(y, x z)=0 .
$$

The notion of pseudo-Riemannian Lie algebras was introduced by Boucetta in [1], which are strongly related to pseudo-Riemannian Poisson manifolds (for more details see $[2])$.

In this note, we focus on pseudo-Riemannian associative fermionic Novikov algebras, which are pseudo-Riemannian fermionic Novikov algebras satisfying

$$
(x y) z=x(y z) \quad \text { for any } x, y, z \in A \text {. }
$$

It is proved in [6] that any pseudo-Riemannian Lie algebra is solvable if the characteristic of $\mathbb{F}$ is zero. For pseudo-Riemannian associative fermionic Novikov algebras, we have:

Theorem 1.1. The underlying Lie algebra of any pseudo-Riemannian associative fermionic Novikov algebra is 2-step nilpotent.

Proof. Let $A$ be a pseudo-Riemannian associative fermionic Novikov algebra and $f$ the corresponding bilinear form. Since $(x y) z=x(y z)$ for any $x, y, z \in A$, we can represent the product only by $x y z$. Furthermore for any $x, y, z, d \in A$,

$$
f(x y z, d)=-f(y z, x d)=f(z, y x d)=-f(y x z, d) .
$$

It follows that $x y z=-y x z$ by the nondegeneracy of $f$. Then we have

$$
x y z=y z x=z x y=-y x z=-z y x=-x z y .
$$


By (1.9), we know that $x y$ belongs to the center of the underlying Lie algebra. It follows that the underlying Lie algebra is 2-step nilpotent.

\section{Pseudo-Riemannian associative fermionic Novikov algebras and Novikov algebras}

A Novikov algebra was introduced as a left-symmetric algebra with commutative right multiplication operators: an algebra is a Novikov algebra if its product satisfies equation (1.1) and

$$
(x y) z=(x z) y \text {. }
$$

It connects with the Poisson brackets of hydrodynamic type [7, 8] and Hamiltonian operators in the formal variational calculus $[11,17]$.

A pseudo-Riemannian Novikov algebra is a Novikov algebra with a nondegenerate symmetric bilinear form satisfying the equation (1.5). It is proved in [5] that pseudo-Riemannian Novikov algebras are fermionic Novikov algebras if the characteristic of $\mathbb{F}$ is not 2. By [3] or [12], the sets of pseudoRiemannian Novikov algebras and pseudo-Riemannian fermionic Novikov algebras are same if $\mathbb{F}=\mathbb{R}$ and the bilinear forms are positive definite. By [18], pseudo-Riemannian fermionic Novikov algebras of dimensions up to 4 over $\mathbb{C}$ are Novikov algebras. Nevertheless,

Remark $2.1([5])$. For dimensions greater than four, we could neither prove that pseudo-Riemannian fermionic Novikov algebras are Novikov algebras nor find a pseudo-Riemannian fermionic Novikov algebra which is not a Novikov algebra.

In the following, we will give a pseudo-Riemannian associative fermionic Novikov algebra which is not a Novikov algebra. Firstly, we establish a theorem.

Theorem 2.2. Let $A$ be a pseduo-Riemannian associative fermionic Novikov algebra over a filed $\mathbb{F}$. If the characteristic of $\mathbb{F}$ is not 2 , then $A$ is 3-step nilpotent.

Proof. Let $A$ be a pseudo-Riemannian associative fermionic Novikov algebra and $f$ the corresponding bilinear form. Denote the product $(x y) z$ only by $x y z$. By the proof of Theorem 1.1, xy belongs to the center of the underlying Lie algebra. Then

$$
x y z d=y z x d=-y z d x=-x y z d .
$$

That is, $x y z d=0$. Namely $A$ is 3-step nilpotent.

Example 2.3. Assume that $A$ is a pseudo-Riemannian associative fermionic Novikov algebra, which is not a Novikov algebra. By [5], we must have $x y z \neq 0$ for some $x, y, z \in A$. By (1.9), we have $x x y=0$ for any $x, y \in A$. If $A$ is algebraically generated by $x, y, z$, then it is easy to see that $x y z \in A^{\perp}$ since $x x y=0$ and $x y z d=0$ for any $x, y, z, d \in A$. It follows that $x y z=0$. 
Assume that $x y z \neq 0$ for some $x, y, z \in A$. Then there exists another element $d$ such that $f(x y z, d)=a \neq 0$. In the following, assume that $A$ is algebraically generated by $x, y, z, d$. Without loss of generality, let $a=1$. By the equation (1.5), we know that $x y d, x z d, y z d$ are not zero and

$$
f(x y z, d)=-f(x y d, z)=-f(y z d, x)=f(x z d, y)=1 .
$$

Let $V_{1}$ be a subspace of $A$ linearly generated by $x, y, z, d$. Furthermore, assume that $u u=0$ for any $u \in V_{1}$. By the linearity of products, we have that

$$
u v=-v u \quad \text { for any } u, v \in V_{1} \text {. }
$$

It is easy to see that $x y, x z, x d, y z, y d, z d$ are not zero. In fact, assume that $x y=0$. Then

$$
f(x y z, d)=f(z x y, d)=-f(x y, z d)=0 .
$$

It is a contradiction. Similar to the others.

Moreover, $x, y, z, d, x y, x z, x d, y z, y d, z d, x y z, x y d, x z d, y z d$ are linearly independent. In fact, assume that there exist $a_{i}$ for $1 \leq i \leq 14$ such that

$$
\begin{aligned}
& a_{1} x+a_{2} y+a_{3} z+a_{4} d \\
& +a_{5} x y+a_{6} x z+a_{7} x d+a_{8} y z+a_{9} y d+a_{10} z d \\
& +a_{11} x y z+a_{12} x y d+a_{13} x z d+a_{14} y z d=0 .
\end{aligned}
$$

Multiplying $x y$ on the left of the above equation, we have

$$
a_{3} x y z+a_{4} x y d=0 .
$$

It follows that

$$
a_{3}=f\left(a_{3} x y z, d\right)=f\left(a_{3} x y z+a_{4} x y d, d\right)=0
$$

since $f(x y d, d)=0$. Similarly, we have $a_{1}=a_{2}=a_{3}=a_{4}=0$. Then the equation is

$$
\begin{aligned}
a_{5} x y+ & a_{6} x z+a_{7} x d+a_{8} y z+a_{9} y d+a_{10} z d \\
& +a_{11} x y z+a_{12} x y d+a_{13} x z d+a_{14} y z d=0 .
\end{aligned}
$$

Multiplying $x$ on the left of the above equation, we have

$$
a_{8} x y z+a_{9} x y d+a_{10} x z d=0 .
$$

It follows that

$$
a_{8}=f\left(a_{8} x y z, d\right)=f\left(a_{8} x y z+a_{9} x y d+a_{10} x z d, d\right)=0 .
$$

Similarly, we have $a_{5}=a_{6}=a_{7}=a_{8}=a_{9}=a_{10}=0$. Then the equation is

$$
a_{11} x y z+a_{12} x y d+a_{13} x z d+a_{14} y z d=0 .
$$

It follows that

$$
a_{11}=f\left(a_{11} x y z, d\right)=f\left(a_{11} x y z+a_{12} x y d+a_{13} x z d+a_{14} y z d, d\right)=0 .
$$


Similarly, we have that $a_{11}=a_{12}=a_{13}=a_{14}=0$. It proves the claim of the linear independence. Also it is easy to get that

$$
f(x y, z d)=f(y z, x d)=-f(x z, y d)=-1 .
$$

In addition putting $f(u, v)=0$ except the eqs. (2.2) and (2.3), we have constructed a pseudo-Riemannian associative fermionic Novikov algebra of dimension 14. It is not a Novikov algebra since $(x y) z=0$ for any $x, y, z \in A$ if $A$ is a pseudo-Riemannian Novikov algebra [5].

Acknowledgments. This work is partially supported by National Natural Science Foundation of China (No. 10971103 and 11001133) and the Fundamental Research Funds for the Central Universities. We would like to acknowledge Professor C. M. Bai for his helpful suggestions and communication with us his research in this field, and the referee for his helpful suggestions and corrections.

\section{References}

[1] M. Boucetta, Poisson manifolds with compatible pseudo-metric and pseudo-Riemannian Lie algebras, Differential Geom. Appl. 20 (2004), no. 3, 279-291.

[2] - Compatibility between pseudo-Riemannian structures and Poisson structures, C. R. Acad. Sci. Paris Sér. I Math. 333 (2001), no. 8, 763-768.

[3] _ On the Riemann-Lie algebras and Riemann-Poisson Lie groups, J. Lie Theory 15 (2005), no. 1, 183-195.

[4] D. Burde, Simple left-symmetric algebras with solvable Lie algebra, Manuscripta Math. 95 (1998), no. 3, 397-411.

[5] Z. Chen and F. Zhu, Pseudo-Riemannian Novikov algebras, J. Phys. A 41 (2008), no. 31, 315207, 9 pp.

[6] _ On pseudo-Riemannian Lie algebra: a class of new Lie admissible algebras, arxiv:0807.0936, 2008.

[7] B. A. Dubrovin and S. P. Novikov, Hamiltonian formalism of one-dimensional systems of hydrodynamic type, and the Bogolyubov-Whitman averaging method, Dokl. Akad. Nauk SSSR 270 (1983), no. 4, 781-785.

[8] _ Poisson brackets of hydrodynamic type, Dokl. Akad. Nauk SSSR 279 (1984), no. 2, 294-297.

[9] I. M. Gel'fand and L. A. Dikii, Asymptotic behaviour of the resolvent of Sturm-Liouville equations and the algebra of the Korteweg-De Vries equations, Russ. Math. Surv. 30 (1975), 77-113.

[10] A Lie algebra structure in the formal calculus of variations, Funktsional. Anal. i Priložen. 10 (1976), no. 1, 18-25.

[11] I. M. Gel' fand and I. Ya Dorfman, Hamiltonian operators and algebraic structures associated with them, Funktsional. Anal. i Prilozhen. 13 (1979), no. 4, 13-30.

[12] J. Milnor, Curvatures of left invariant metrics on Lie groups, Advances in Math. 21 (1976), no. 3, 293-329.

[13] A. A. Sagle, Nonassociative algebras and Lagrangian mechanics on homogeneous spaces, Algebras Groups Geom. 2 (1985), no. 4, 478-494.

[14] E. B. Vinberg, The theory of convex homogeneous cones, Trudy Moskov. Mat. Obshch. 12 (1963), 303-358.

[15] X. Xu, Hamiltonian superoperators, J. Phys. A 28 (1995), no. 6, 1681-1698.

[16] _ Variational calculus of supervariables and related algebraic structures, J. Algebra 223 (2000), no. 2, 396-437. 
[17] _ Hamiltonian operators and associative algebras with a derivation, Lett. Math. Phys. 33 (1995), no. 1, 1-6.

[18] F. Zhu and Z. Chen, Bilinear forms on fermionic Novikov algebras, J. Phys. A 40 (2007), no. 18, 4729-4738.

ZHIQI CHEN

School of Mathematical Sciences and LPMC

NANKAi UnIVERSITY

Tianjin 300071, P. R. China

E-mail address: chenzhiqi@nankai.edu.cn

FuHAi ZHU

School of Mathematical Sciences and LPMC

NANKAi UNIVERSITY

Tianjin 300071, P. R. China 